\title{
MedienPädagogik
}

Zeitschrift für Theorie und Praxis der Medienbildung

Jahrbuch Medienpädagogik 18: Ästhetik - Digitalität - Macht

Herausgegeben von Benjamin Jörissen, Claudia Roßkopf, Klaus Rummler,

Patrick Bettinger, Mandy Schiefner-Rohs, Karsten D. Wolf

\section{Choreografien vor den Überwachungskameras der Kleinstadt}

\author{
Selbstaufnahmen und digitale Assemblagen in \\ (SELFIE2) von Kılınçel \& Schaper als Befragung von \\ Machtfigurationen in ländlichen Räumen
}

\author{
Barbara Sterzenbach $^{1} \bullet$, Micha Kranixfeld ${ }^{1} \bullet$ und Wiebke Waburg ${ }^{1} \bullet$ \\ ${ }^{1}$ Universität Koblenz-Landau
}

\section{Zusammenfassung}

Im Jahr 2020 stellten die Künstlerinnen und Künstler Kılınçel \& Schaper sich und der Kleinstadt Schotten die Aufgabe, ein Selfie zu produzieren, welches die Gemeinde abbilden sollte. Im Verlauf entstanden vielfältige Begegnungen und Kollaborationen mit der Bevölkerung, die schliesslich auf einer Website zu einer digitalen Assemblage zusammengefügt wurden. Der Beitrag kombiniert die Analyse des partizipativ hervorgebrachten digitalen Kunstwerks mit ethnografischen Beobachtungen des Produktionsprozesses, der ebenfalls auf verschiedenerlei Weise von Digitalität geprägt war. Dabei fokussieren die Autorinnen und Autoren auf die Arbeit mit Jugendlichen aus der Gemeinde. Im Rückgriff auf Machtbegriffe von Imbusch sowie Deleuze und Guattari wird untersucht, wie Selbstaufnahmen und digitale Assemblagen Machtfigurationen in ländlichen Räumen befragen und verschieben können. Es konnte herausgearbeitet werden, dass das partizipativ produzierte digitale Kunstwerk nicht ohne Blick auf die machtvollen sozialen Prozesse verstanden werden kann, die zu seiner Herstellung geführt haben. Dabei konnte aufgezeigt werden, dass sozialer und künstlerischer Prozess als gleichberechtigte Teile einer gegenseitigen kulturellen Bildung erscheinen, die 
Künstlerinnen, Künstler und Teilnehmende gemeinsam durchlaufen. Mit ihrer Arbeit agieren Kılınçel \& Schaper temporär in einem lokalen Jugendzentrum und sind herausgefordert, sich innerhalb persönlicher, pädagogischer, machtvoller und machtkritischer sowie künstlerisch-ästhetischer Spannungsfelder zu verorten. Den Jugendlichen eröffnet sich mithilfe künstlerischer Verfahrensweisen, die digitalen und physischen Raum verbinden, ein erweitertes, kreatives Handlungsspektrum im Umgang mit der Überwachung ihrer Räume.

\title{
Choreographies in Front of Small Town Surveillance Cameras. Self-Photography and Digital Assemblages in 〈SELFIE2) by Kilınçel \& Schaper as an Interrogation of Power Figures in Rural Spaces
}

\begin{abstract}
In 2020, the artists Kllinçel \& Schaper set themselves and the small town of Schotten the task of producing a selfie that would depict the community. In the process, a variety of encounters and collaborations with the population emerged, which were finally combined into a digital assemblage on a website. The article combines the analysis of the participatory digital artwork with ethnographic observations of the production process, which was also characterized by digitality in various ways. The authors focus on the work with young people from the community. Drawing on concepts of power from Imbusch as well as Deleuze and Guattari, they explore how self-recordings and digital assemblages can interrogate and shift figures of power in rural spaces. The authors assume that the participatory produced digital artwork cannot be understood without regarding the powerful social processes that led to its production. In doing so, it was possible to work out that social and artistic processes appear as equal parts of a mutual cultural education that artists and participants go through together. With their work, Kllınçel \& Schaper operate temporarily in a local youth center and are challenged to situate themselves within personal, pedagogical, power-critical, and artistic-aesthetic fields of tension. With the help of artistic procedures that combine digital and physical space, the young people open up an expanded, creative spectrum of action in dealing with the surveillance of their spaces.
\end{abstract}




\section{Einleitung: Ein Selfie der Gemeinde Schotten}

Ein sonniger Tag im September 2020 in Schotten, einer Kleinstadt in Hessen. Eine Überwachungskamera filmt die Ecke eines Hauses. Um 15:49 Uhr zeichnet sie eine junge Frau auf, die aus dem Haus kommt und ein Kehrblech in der Hand hält. Sie bleibt stehen und lässt ihren Arm nach oben schnellen. Der Inhalt des Kehrblechs rieselt als grosse weisse Wolke auf den Boden (vgl. Abb. 1).

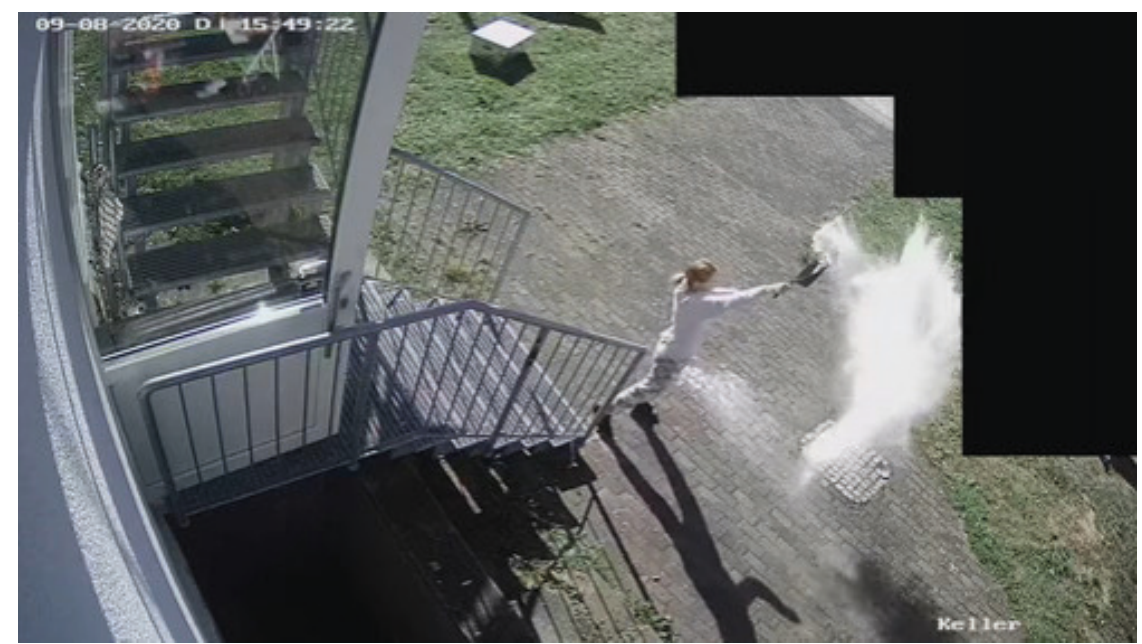

Abb. 1: Screenshot des Videos https://schotten2020.de/video/fajuso/FaJuSo_ Ausgang.mp4; 00:23 von 02:03 Min. am 21.01.2022.

Diese bemerkenswerte Szene findet sich als Videoclip auf der Website www.schotten2020.de, einem digitalen Kunstwerk von Kllınçel \& Schaper. In ihrem Projekt stellten sie die Frage, wie das «Selfie einer Gemeinde» (FLUX - Netzwerk Theater und Schule) aussehen könnte. Dafür sammelten und inszenierten sie über einen Zeitraum von vier Monaten mosaikartig Eindrücke der Kleinstadt Schotten gemeinsam mit deren Bewohnerinnen und Bewohnern:

«Aus den Begegnungen ergaben sich die Inhalte dieser Website. Die Bewohner:innen in Schotten haben durch Hilfestellung oder aktiv mit eigenen Beiträgen dazu beigetragen. Entstanden ist daraus eine digitale Collage, ein Stadt-Selfie, irgendwo zwischen Portrait und Reiseführer, eine Momentaufnahme ohne Anspruch auf Vollständigkeit.» (Kılınçel und Schaper 2020) 
Die Videosequenz mit der jungen Frau, die ihr Kehrblech ausschüttet, ist nur eines von vielen Elementen der Seite. Sie entstand während eines mehrtägigen Videoprojektes, welches in Kooperation mit einem lokalen Jugendzentrum durchgeführt wurde, und konnte im Rahmen des Forschungsprojekts $\langle\mathrm{DO}$ _KiL $\rangle$ ethnografisch beobachtet werden. Im vorliegenden Artikel nehmen wir diesen Moment des künstlerischen Arbeitens mit Jugendlichen exemplarisch in den Blick und stellen ihn anhand von sechs selbst beobachteten Szenen dar (4.). Dabei interessiert uns, wie Selbstaufnahmen und digitale Assemblagen Machtfigurationen in ländlichen Räumen befragen und verschieben können. Wir gehen dabei davon aus, dass das partizipativ produzierte digitale Kunstwerk nicht ohne die machtvollen sozialen Prozesse verstanden werden kann, die zu seiner Herstellung geführt haben. Der empirischen Analyse vorangestellt sind eine Vorstellung des Forschungsprojektes 〈DO_KiL〉(2.) sowie des Kunstprojekts 〈SELFIE2〉 der Residenzkünstlerinnen und Residenzkünstler (3.). Dies zielt darauf, einerseits Forschungsgegenstand, Forschungsfeld und methodisches Vorgehen des Forschungsprojekts vorzustellen, andererseits den Kontext der Durchführung des Videoprojekts - in Form einer partizipativ ausgerichteten Erstellung eines digitalen Selfies einer Gemeinde - zu verdeutlichen (3.).

\section{Das Forschungsprojekt (DO_KiL)}

Das Projekt 〈DO_KiL〉 (Der Dritte Ort? Künstlerische Residenzen in ländlichen Räumen) ${ }^{1}$ der Universität Koblenz-Landau untersucht im Sinne einer Grundlagenforschung temporär angelegte Residenzen in ländlichen Räumen als Spiel-, Versammlungs- und Kommunikationsorte. Eine Residenz ermöglicht es den Künstlerinnen und Künstlern für eine bestimmte Zeit, an einem bestimmten Ort - dem Residenzort - zu einem selbstgewählten Thema zu arbeiten und zu forschen (vgl. Generaldirektion der Europäischen

1 Unter der Projektleitung von Wiebke Waburg, der wissenschaftlichen Mitarbeit von Micha Kranixfeld und Barbara Sterzenbach sowie in Kooperation mit Kristin Westphal und Ilona Sauer. Das Projekt wird gefördert durch das Bundesministerium für Bildung und Forschung (BMBF). Die Projekt-Webseite kann hier besucht werden: https://www.uni-koblenz-landau.de/de/koblenz/ $\mathrm{fbl} / \mathrm{sempaed} / \mathrm{migration/forschungsprojekte/aktuelle-forschungsprojekte.}$ 
Kommission für Bildung, Jugend, Sport und Kultur 2016). Dabei wird meist davon ausgegangen, dass als Residenzort eine Gemeinde fungiert, in der diese sonst nicht leben. Die Begegnung von Künstlerinnen bzw. Künstlern und Bevölkerung wird von Residenzprogrammen meist als wechselseitige Herausforderung konzipiert (vgl. Waburg et al. 2022).

Künstlerische Residenzen werden im Projekt $\langle\mathrm{DO}$ _KiL〉 nicht nur in Bezug auf die künstlerische Arbeit und die Spuren, die sie hinterlassen, sondern auch daraufhin untersucht, wie sie als Formate kultureller Bildung in ländlichen Räumen konzipiert und umgesetzt werden (ebd.). Damit wird ein bestehendes Forschungsdesiderat adressiert, da empirische Untersuchungen kultureller Bildungsangebote und -projekte in ländlichen Räumen bislang kaum vorliegen. Das Projekt fusst auf einem breiten Verständnis von kultureller Bildung, die als «Allgemeinbildung durch spezifische ästhetische Erfahrungen definiert» wird (Rat für Kulturelle Bildung 2014, 14f.). Studien zur kulturellen Bildung in urbanen Räumen belegen, dass Kinder und Jugendliche aus bildungsbenachteiligten Familien selten mit den Künsten in Kontakt kommen (vgl. Rat für Kulturelle Bildung 2015), dies gilt ebenfalls für Heranwachsende aus Familien mit Migrationsgeschichte - für die ebenfalls zu konstatieren ist, dass die Partizipation am Kulturleben vom sozialen Hintergrund beeinflusst wird (vgl. Mandel 2016). Es ist vorstellbar, dass sich im ländlichen Raum ähnliche Muster zeigen. Dies wäre insofern problematisch, da

«Kulturelle Bildung [...] als ein unverzichtbarer Beitrag zur Persönlichkeitsentwicklung betrachtet [wird], der vor dem Hintergrund einer globalisierten und zunehmend digitalisierten Welt für Kinder und Jugendliche an Bedeutung gewinnt, um den gesellschaftlichen Herausforderungen, z. B. dem Umgang mit kultureller Vielfalt, begegnen zu können.» (Keuchel und Werker 2018, 1)

Dementsprechend ist davon auszugehen, dass kulturelle Bildung Teilhabechancen von Kindern, Jugendlichen und Erwachsenen mit unterschiedlichen Hintergründen eröffnet (Kolleck und Büdel 2020) und zu ihrem Empowerment beitragen kann. 
Ob und wie dies im Rahmen von Residenzprojekten geschieht - in denen Künstlerinnen und Künstler aufgerufen sind, den Teilnehmenden die Möglichkeit zu eröffnen, «künstlerisch ästhetische Erfahrungen zu machen» (ebd.) $)^{2}$-, wird im Projekt 〈DO_KiL〉 untersucht. Den Forschungszugang bilden ethnografische Beobachtungen, Gespräche und Interviews mit den Kunstschaffenden und Teilnehmenden aus dem Ort sowie die Analyse der entstandenen Kunstwerke (Aufführungen, Filme, Interventionen). Die Auswertung erfolgt mittels dokumentarischer Methode (vgl. Bohnsack, Nentwig-Gesemann und Nohl 2013). Die im Rahmen des vorliegenden Artikels präsentierten Ergebnisse basieren vor allem auf der Beschreibung des Projekts, des in ihm entstandenen digitalen Kunstwerks (siehe 3.) und der sequenziellen Analyse von Beobachtungsprotokollen (siehe 4.). Letztere wurden zunächst formulierend interpretiert. Dabei lag der Fokus auf dem Beschreiben dessen, was passiert und somit dem immanenten Sinngehalt. Die sich anschliessende reflektierende Interpretation zielte auf die Erarbeitung des dokumentarischen Sinngehalts, also des atheoretischen Wissens, das reflexiv meist nicht zugänglich ist, jedoch der Handlungspraxis der Akteurinnen und Akteure zugrunde liegt.

\section{Das künstlerische Projekt: «SELFIE2» (2020) von Kılınçel \& Schaper}

Das Selfie einer Gemeinde zu produzieren - diese Aufgabe stellten Kılınçel \& Schaper sich und der Kleinstadt Schotten. Dabei handelte es sich um eine Fortsetzung der Residenz aus dem Vorjahr, in der Jugendliche ihre Selfies mithilfe verschiedener Techniken dekonstruierten. Für Jugendliche sind Selfies ein alltägliches Phänomen besonders innerhalb digitaler sozialer Netzwerke und heutiger Medienkultur. «Wer ein Selfie macht, macht sich selbst zum Bild» (Ullrich 2019, 6). Mit der Setzung, ein Selfie der Gemeinde zu produzieren, entwickelten die Künstlerinnen und Künstler eine Herausforderung, der sich alle Menschen aus der Gemeinde gemeinsam mit ihnen stellen konnten: Wie könnte aus den vielen Singulären ein Selfie entstehen?

2 Häufig fehlt den Künstlerinnen und Künstlern eine entsprechende pädagogische Qualifikation (Keucher und Werker 2018, 1). 
Wer und was müsste zu sehen sein, damit das Abbild für die ganze Gemeinde stehen kann? Die Antwort lag in den Möglichkeiten digitaler Programmierung.

Kilınçel \& Schaper legten das Projekt zunächst als künstlerische Feldforschung an, bei der sie unterschiedlichste Orte und Initiativen der Gemeinde besuchten - beispielshaft die Freiwillige Feuerwehr, einen Dudelsackspieler oder die Nachbarschaftshilfe. Sie dokumentierten die Besuche durch Selfies auf Instagram ${ }^{3}$ und erarbeiteten in einem zweiten Schritt mit einigen dieser Kontakte Beiträge für die Website. Physische Begegnung und reale Repräsentation fanden sich damit in einem dauernden Wechselverhältnis. Das abschliessende Arrangement der Beiträge lag - auch aufgrund der Corona-Pandemie - in den Händen der Künstlerinnen und Künstler, die Umsetzung in denen einer Webdesignerin und der ihr zur Verfügung stehenden digitalen Tools. Die Autorinnen- und Autorenschaft lag damit nicht allein bei den Kunstschaffenden, sondern zeigte sich «als verteilter, netzwerkartiger und vor allen Dingen nicht vollständig begreifund bestimmbarer Prozess» (Herlitz und Zahn 2019), ${ }^{4}$ in dem keine der beteiligten Personen die vollständige Kontrolle innehatte. In der entstandenen Website spiegelt sich dieser Prozess wider: Um die einzelnen Elemente zu sehen, muss man in alle Richtungen scrollen; ein Gesamtbild gibt es nicht (vgl. Abb. 2, bei der trotz der Verkleinerung der Inhalte auf 30 \% nur ein kleiner Teil im Browser erscheint). Zudem werden die Elemente (Fotogalerien, handschriftliche Notizen, Interviewfragmente und Videos) mit jedem Aufruf der Seite neu angeordnet, sodass sie sich nur schwer gezielt wiederfinden lassen. Die digitale Assemblage ${ }^{5}$ bleibt beständig im Wandel.

3 https://www.instagram.com/_s_e_l_f_i_e_s/.

4 Herlitz und Zahn entwickelten einen Analyserahmen für bildungstheoretische Potenziale postdigitaler Ästhetiken, der sich in Teilen auch für unsere Analyse als geeignet erwies, besonders im Hinblick auf verteilte Autorinnenund Autorenschaft und Übergänge zwischen virtuellen und physischen Räumen.

5 Der Begriff der Assemblage bezeichnet im Bereich der Bildenden Künste besondere Collagen: Kombinationen verschiedener (plastischer) Gegenstände, Materialien, Bruchstücke, die auf einem Untergrund arrangiert werden. Im künstlerischen Arrangement werden die zunächst unabhängigen Dinge in Beziehung zueinander gesetzt (vgl. Seitz 1961, 6). 


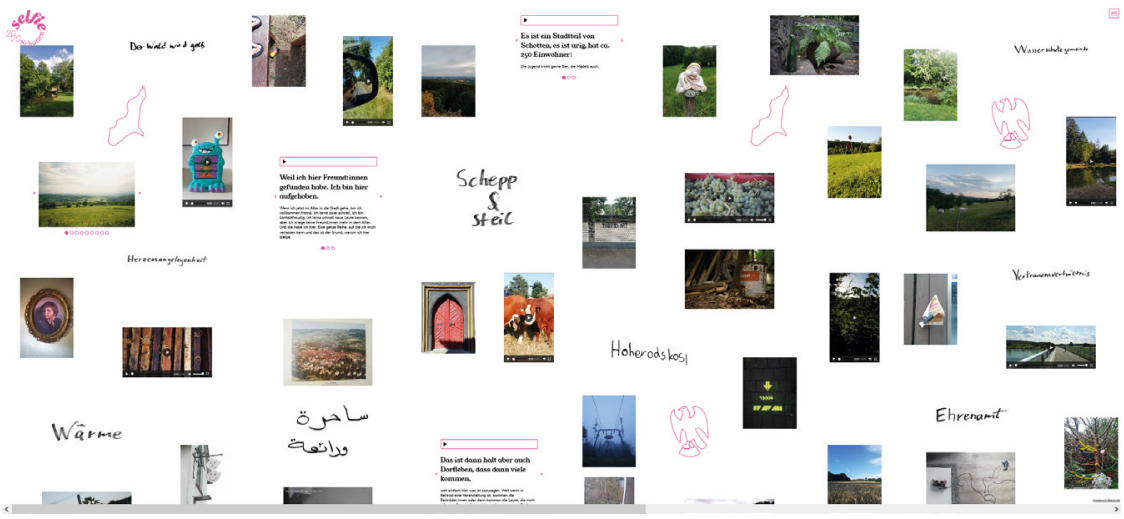

Abb. 2: Screenshot der Webseite schotten2020.de am 21.01.2022 (Zoomfaktor 30\%).

Kilınçel \& Schaper verzichten auf die detaillierten Einordnungen des Materials und fordern die Betrachtenden heraus, Konstellationen und Bezüge für sich selbst herzustellen. Dies ist vielleicht am ehesten für Menschen aus Schotten möglich. Sie (er)kennen das Abgebildete, Ortsteile und Landschaften in ihren vielfachen Verweisungszusammenhängen und die damit verbundene machtvolle Ordnung. Im Abgleich von eigener Ortskenntnis und digitalen Neuarrangements reflektieren sie beim Betrachten der Website «Stellen des Übergangs» (Herlitz und Zahn 2019) von digitalen und physischen Räumen. Man kann die Form der Website darum auch als Antwort auf die selbstgestellte Aufgabe interpretieren: Ihr dynamischer Aufbau verweist darauf, dass ein 〈Selfie〉 der Kleinstadt Schotten nur aus einer Vielzahl teils widersprüchlicher Selbstbilder zusammengefügt werden kann, die wiederum auf weitere Zusammenhänge verweisen. Das 〈Selfiè einer Kommune, wie es Kllınçel \& Schaper entwerfen, versucht kein homogenisierendes Abbild, sondern legt im Prozess seiner Entstehung die vielfältigen Schichten frei, die ein 〈Selfie〉 von Schotten enthalten könnte. Das Kunstwerk interveniert damit u. a. in Selbsterzählungsmuster kleiner Gemeinden, die nach Identischem und Homogenem suchen. Damit rückt der Sozialraum als wesentlicher Kontext bei der Betrachtung des digitalen Kunstwerks in den Blick. 


\section{Künstlerisches Handeln als Intervention in Machtfigurationen}

In Anbetracht der angesprochenen Vielzahl von Bedeutungskontexten der entstandenen Assemblage fokussieren wir für diesen Artikel auf die mit Jugendlichen aus Schotten entwickelten Videofragmente vor Überwachungskameras. Die beteiligten Jugendlichen nehmen innerhalb der Gemeinde eine marginalisierte Position ein und werden digital überwacht (siehe 4.1). An ihrem Beispiel lassen sich Zusammenhänge von Digitalität, Kultureller Bildung und Sozialraum exemplarisch betrachten.

Im Rückgriff auf Feldnotizen rekonstruieren wir den Arbeitsprozess von Kilınçel \& Schaper. In diesem Kontext interessiert uns besonders, wie die Idee der digitalen Selbstaufnahme als Befragung von Machtfigurationen in ländlichen Räumen wirksam werden kann und auf welche Weise die Künstlerinnen und Künstler durch ihre Residenz und die entstehende digitale Assemblage in diese lokalen Machtfigurationen hineinwirken. In Anlehnung an Imbusch verstehen wir unter Machtfigurationen:

«ein komplexes Geflecht asymmetrischer und wechselseitiger Beziehungen, in dem mehrere Personen, Gruppen oder Parteien miteinander verknüpft sind und in dem Veränderungen einer Relation auch die anderen Relationen ändern [...]. Das Konzept der Machtfiguration erlaubt eine genuin soziologische Analyse. Es rekonstruiert die Dynamik von Machtprozessen [...] aus dem sozialen Verhältnis selbst.» (Imbusch 2007, 397)

Diese Perspektive erlaubt eine fluide Sicht auf Machtprozesse, wie sie auch im (Assemblage)-Begriff von Deleuze und Guattari durch die Begriffe 〈De- und Reterritorialisierung〉 angelegt wird. Deterritorialisierung und Reterritorialisierung meinen dabei zirkuläre Auflösungs- und Neuordnungsprozesse von Beziehungsgefügen jeglicher Art (vgl. Deleuze und Guattari 1987, 10, 381). Da Künstlerinnen und Künstler im Rahmen einer Residenz zwangsläufig innerhalb lokaler Machtfigurationen agieren, wäre folglich zu beobachten, auf welche Weise ihre Arbeit (de)stabilisierend für die Gefüge wirkt und inwiefern sie mit ihrer Kunst neue Prozesse anstossen können. 


\subsection{Das Jugendhilfezentrum als Partner der Künstlerinnen und Künstler und als Akteur lokaler Machtfigurationen}

In einem Gespräch zu Beginn der ethnografischen Begleitung berichteten Kllınçel \& Schaper, dass ihnen bereits im Vorjahr bei Spaziergängen durch Schotten die Überwachung öffentlicher Plätze mit Kameras sowie eine problematisierende Einstellung gegenüber einigen Jugendlichen des Ortes aufgefallen war, die von vielen Kontaktpersonen als delinquent oder unangepasst beschrieben wurden (vgl. Interview mit Kılınçel \& Schaper, GI-03-RKo03-20200708, Z. 89-93). Einteilungen in «gute» (meint meist: in Vereinen aktive und höhere Bildungsabschlüsse anstrebende) und «schlechte» (meint: nicht institutionell eingebundene, Zwanglosigkeit suchende und weniger hohe Bildungsabschlüsse machende) Jugendliche sind keine Eigenart ländlicher Räume. Sie erfahren hier aber besondere Brisanz, denn mit dem Blick auf die Jugendlichen geht auch eine Bewertung der Familien und der Lebenschancen einher, die durch die engeren Netzwerke in ländlichen Gemeinden langfristige Auswirkungen haben (vgl. Eckardt 2018, 196ff.).

Auch an dem Jugendzentrum, mit dem Kılınçel \& Schaper für ihr Projekt kooperieren, waren Überwachungskameras installiert, die ihre digitalen Bilder an einen anonymen, von der Kommune beauftragten Dienstleister übermitteln. Die digitale Überwachung von Aufenthaltsräumen von Jugendlichen mit Kameras kann als «gemeinschaftliche[s] Sicherheitshandeln» (Oelkers und Schierz 2019, 99) beschrieben werden, in dem sich behördliche Schutzmassnahmen und ländliche Sozialkontrolle verschränken. Die staatliche Kontrolle steht im Konflikt mit der Raumaneignung Jugendlicher, die sich ihre Bühnen und Nischen schaffen, wodurch diese Orte zu Räumen der Selbstdarstellung, der Identitätsfindung und der Abgrenzung zu anderen Gruppen werden (vgl. Bauer 2020, 26). Das Jugendzentrum folgt entgegen der Überwachungslogik einer sozialräumlichen Orientierung, die zum Ziel hat,

«sozialräumliche Zusammenhänge derart zu arrangieren, dass sie 〈bildsam〉 sind, dass sie also Aneignungsprozesse fördern und damit Jugendlichen Handlungsoptionen, gesellschaftliche Teilhabe und biografische Perspektiven eröffnen.» (Deinet und Krisch 2021, 1064) 
Dieses Verständnis von Bildungsprozessen trifft sich nun mit der Förderbedingung der künstlerischen Residenz von Kılınçel \& Schaper, im Rahmen ihrer Arbeit in Schotten kulturelle Bildungsprozesse anzuregen (vgl. FLUX - Netzwerk Theater und Schule o. J.).

Im Folgenden werden nun sechs Szenen einer teilnehmenden Beobachtung herangezogen, um die komplexen Bedingungen darzustellen, unter denen in partizipativen künstlerischen Projekten mit Jugendlichen Machtfigurationen zwischen verschiedenen Akteurinnen und Akteuren befragt werden können.

\subsection{Sechs Szenen des künstlerischen Prozesses}

\subsubsection{Szene 1: Bildungsauftrag}

Die angereiste Beobachterin kennt sich in Schotten nicht aus. Darum zeigt ihr die Künstlerin Tümay Kılınçel, ebenfalls zu Gast im Ort, worum es im Folgenden gehen soll:

«Das ist das [Name des Jugendzentrums]》, dann zeigt sie mit dem Finger nacheinander auf drei Punkte an der Hauswand, «da sind überall Überwachungskameras. Das gibt es sonst nirgendwo in der Stadt, so eine starke Überwachung. Nur hier am [Jugendzentrum]〉, sagt sie. Eine Kamera ist rechts neben der Wendeltreppe angebracht, zwei weitere sind links am Haus angebracht. Eine guckt eine Treppe hinab zum Kellereingang, die andere erfasst wohl den Eingangsbereich und den kurzen Treppenaufgang.» (Feldnotiz BS_09_003_20200908,1-2)

Hier werden 〈De- und Reterritorialisierungsprozesse〉 eingeleitet: Die Überwachung des Hauses erscheint der Künstlerin als Machtinstrument staatlicher/öffentlicher Akteurinnen und Akteure, um die Handlungsmöglichkeiten der Jugendlichen einzuschränken, die im Ort als kaum zu kontrollieren und problematisch gelten. Die Künstlerin scheint mit dem Hinweis auf die Ungleichbehandlung (〈nur hier〉) eine Stigmatisierung der Jugendlichen belegen zu wollen. Die Kameras besetzen stellvertretend für 
körperlose Dritte den Raum der Jugendlichen. Der Voyeurismus des Zusehens und Aufzeichnens bereitet eine visuelle Beweisführung gegen antizipierte Handlungen vor, die von ebenjenen Unbekannten als unangebracht oder strafbar definiert werden. Dieser Voyeurismus wird jedoch in dem Moment deterritorialisiert, in dem auf die 〈Verstecke〉 hingewiesen wird. Nun ist es möglich, der Überwachung zu entgehen, sich ihr zu verweigern oder sie ins Absurde zu führen (Reterritorialisierung). Aus einem passivem Beobachtet-werden kann nun eine aktive Begegnung entstehen. Ausgehend von ihren Beobachtungen hat die Künstlerin ein Videoprojekt für die Nutzenden des Jugendzentrums entworfen:

«[Tümay erklärt] mir die Ideen, die sie für die Alltagsperformance hat. Man könnte Körperteile am Rand des Bildausschnitts verstecken, Regenschirme demonstrativ aufspannen, posieren wie ein Model und die Kamera blenden mit Taschenlampen.»(Feldnotiz BS_09_003_20200908, 2)

Ein Austausch mit den Jugendlichen hat zu diesem Zeitpunkt noch nicht stattgefunden. Die Überlegungen zur Gestaltung des Videoprojekts orientieren sich einerseits an ihren Erfahrungen als Tänzerin mit Inszenierungen des menschlichen Körpers im öffentlichen Raum, andererseits an (begründeten und unbegründeten) Vorannahmen über die Ausdrucksmöglichkeiten der ihr bis dahin fremden Jugendlichen.

Durch die thematischen Setzungen der Künstlerinnen und Künstler sollen Phänomene digitalisierter (Jugend)Räume angesprochen werden: die Auseinandersetzung mit digitalen Selbstinszenierungen durch Selfies sowie die problematisierte digitalisierte Überwachung durch Kameras an öffentlichen Plätzen (vgl. Kemper und Reutlinger 2015). So zielen ihre Ideen auf eine reflektierte Rückeroberung (Reterritorialisierung) des überwachten eigenen Körpers durch eine Umkehrung des Blicks ab. Eine physische Selbstermächtigung soll hier ermöglicht werden, indem Körperteile bewusst und kontrolliert den Kameras preisgegeben werden oder vor ihnen versteckt bleiben. Für die potenziellen Beobachtenden an einem unbekannten Ziel der Kameraübertragung entspricht dies einer Störung der beauftragten Überwachung. In der Vorstellung der Künstlerin wird die 
Überwachung durch das bewusste Spiel mit ihr ausgehebelt. Ihr eigenes Interesse, den Voyeurismus der Überwachenden zu kritisieren, setzt sie jedoch bei den Jugendlichen nicht als selbstverständlich voraus: Später

«[...] erzählt Tümay von ihrer Idee, den Jugendlichen erst ein Musikvideo zu zeigen, um sie zu motivieren. (Vielleicht haben die dann mehr Bock?), fragt sie laut.» (Feldnotiz BS_09_003_20200908, 2)

Die Künstlerin bringt ihre Sorge zum Ausdruck, die Jugendlichen, die keine Beziehung oder Verpflichtung ihr gegenüber haben, nicht für das künstlerische Projekt begeistern zu können oder sie zu überfordern. So offenbart ihre Frage Befürchtungen eines antizipierten Widerstandes gegen sie als (die Andere), ihre professionellen Erfahrungen in ähnlichen Settings und ihre autobiografischen Erfahrungen in dieser besonderen Altersspanne. Auch hier werden De- und Reterritorialisierung sichtbar, indem sich die Künstlerin empathisch in die Jugendlichen hineinversetzt, um deren Bedürfnisse und Vorlieben zu antizipieren, dies jedoch vor dem Hintergrund ihrer eigenen Erfahrungen und Vorstellungen tun muss, da sie die Jugendlichen noch nicht kennen gelernt hat.

\subsubsection{Szene 2: Polizei}

Bei der gemeinsamen Ankunft von Künstlerin, Künstler und Beobachterin an der Einrichtung rückt zunächst eine polizeiliche Vorladung eines Jugendlichen in den Vordergrund. Gemeinsam sprechen Personal und ein weiterer Junge mit dem Betroffenen. Eine Jugendliche $\left(\mathrm{Claire}^{6}\right)$ entzieht sich lautstark dem krisenhaften Gespräch. Die Leiterin des Jugendzentrums und der Vorgeladene begeben sich anschliessend zur Polizeiwache. Kllınçel \& Schaper warten währenddessen mit der Beobachterin in einem angrenzenden Raum auf eine Entscheidung, ob der Workshop noch stattfinden kann.

6 Die Namen der Jugendlichen wurden anonymisiert. 
«Dann kommt einer der Jugendlichen (Chris) rüber: 〈Ich weiß nicht, ob ihr noch was machen wollt? Sind halt nicht mehr so viele da...), schulterzuckend bleibt er in der Tür stehen. 〈Doch klar, wir haben Bock〉, sagt Tümay und der junge Mann holt die anderen.» (Feldnotiz BS_09_003_20200908, 3)

In der einschränkenden Frage des Jugendlichen (später stellt sich heraus, dass er Praktikant ist) spielt nicht nur Unsicherheit zum weiteren Ablauf des Nachmittags eine Rolle, sondern möglicherweise auch eine bestimmte Vorstellung von Filmdrehs, die oft zeitlich gut durchgeplant und organisiert sind. Die lange Wartezeit ist ihm sichtlich unangenehm, was er durch die vorsichtige Formulierung, die nachgeschobene angedeutete Erklärung und die Körpersprache zum Ausdruck bringt: Vielleicht lohnt es sich nicht mehr, jetzt anzufangen? Vielleicht sind die Jugendlichen zu erschöpft (emotional)?

Die Verantwortung, die ihm kurzfristig übertragen wurde - die Leitung ist schliesslich nicht mehr da (Deterritorialisierung) - überträgt er nun auf die erwachsenen Künstlerinnen bzw. Künstler; zumindest temporär werden Kilınçel \& Schaper nun Teil der Assemblage und ihrer hierarchischen Strukturen (Reterritorialisierung). Situativ werden durch die Anwesenheit des Residenzteams und der Beobachterin Hierarchiemuster abgerufen und angeboten, gestützt durch Vorstellungen zu Altersstruktur, Ausbildung/Studium und Rolle als Workshop-Leitungen. «Ob ihr noch was machen wollt» kann hier die Entscheidung an die Angesprochenen abgeben, die Auslassung einer expliziten Benennung, «was» denn gemacht werden solle, verweist auf fehlende Vorstellungen von Themen und Arbeitsweisen der Künstlerinnen bzw. Künstler.

\subsubsection{Szene 3: Skepsis}

«Ein Jugendlicher (Thomas) schlurft grußlos herein und lässt sich neben der Couch auf einen Stuhl plumpsen. Weit genug weg, um nicht in den Laptop schauen zu können oder zu müssen. Chris setzt sich ebenfalls dazu und kündigt noch Kim an, die in der Küche noch etwas aufräumt. Das blonde Mädchen von eben (Claire) setzt sich 
mit den Worten 〈Ich mach aber nicht mit〉 ebenfalls auf die Couch, zieht die Knie an und beschäftigt sich mit ihrem Handy.» (Feldnotiz BS_09_003_20200908,3)

Die Jugendlichen scheinen von der vorhergehenden Situation emotional belastet. Die Vorladung eines anderen durch die Polizei scheint sie auch persönlich betroffen zu machen. Wie blicken sie in diesem Moment auf die Künstlerin und den Künstler? Sind diese nun einfach nachgerückt in eine Position der Kontrolle (Deterritorialisierung)? Zumindest sind Kılınçel \& Schaper nicht mehr unvoreingenommen. Die körperliche und emotionale Erschöpfung sowie die Frustration darüber, (ungewollt) ein Bild vermittelt zu haben, gegen das die Jugendlichen vermutlich oft ankämpfen, ist im Raum spürbar. Ihre abweisende und desinteressierte Haltung kann in diesem Sinne als Selbststabilisierung der Jugendlichen interpretiert werden (Reterritorialisierung), die sich ihre Definitionshoheit (Nicht-Begrüssung der 〈Fremden〉), ihre Räume (dabeisitzen, aber ignorieren) und die Kontrolle ihrer Freizeitgestaltung (Ablehnung der Teilnahme) erhalten möchten. Die Jugendlichen sind aber auch neugierig, wollen zuhören, dabei sein und vielleicht auch umworben werden, schliesslich würden sie sich sonst ganz entziehen. Um das Eis zu brechen, zeigt Tümay Kılınçel drei Videos von Choreografien in öffentlichen Räumen und erklärt, dass sie gerne ähnliche Videos produzieren würde.

«Ja, also wir würden dann mit euch so einen Film machen. (...) Wir würden zum Beispiel hier mit Geländerrutschen anfangen. Das wäre dann auch schon die erste Choreografie), überlegt Tümay laut. Kim kommt hinzu. Sie hat dunkle Haare mit pinkgefärbten Spitzen und ist komplett schwarz gekleidet. Auch sie scheint mit ihren etwa 20 Jahren eher zu den Mitarbeitenden zu gehören. «Was für ein Kindergarten, ey〉, meldet sich Claire zu Wort und lacht laut auf. (...) Kim wird kurz eingeweiht: «Wir würden halt so Alltagssachen dekonstruieren. Handlungen runterbrechen und das von den Sicherheitskameras filmen lassen〉, erklärt Tümay. Dann gehen wir gemeinsam raus, nur Claire bleibt auf der Couch sitzen.» (Feldnotiz BS_09_003_20200908, 3-4) 
In der Nachbetrachtung fällt das Code-Switching der Künstlerin auf. In der Erklärung für die Jugendlichen nutzt sie Filme als Anschauungsmaterial und eine alltagsnahe Sprache. In der Adressierung der Mitarbeiterin Kim greift sie auf akademische Sprache («dekonstruieren») zu und nennt auch die Überwachungskameras als konzeptionellen Bezugspunkt. Dieser Wechsel verdeutlicht Hierarchien: Die Mitarbeiterin wird eher als ebenbürtig angesehen, die Jugendlichen benötigen aus Sicht der Künstlerin mehr Vermittlungsleistung. Gleichzeitig ist dies eine Komponente, die - in Kombination mit der vorgeschlagenen Aktivität des Geländerrutschens bei Claire den Eindruck entstehen lässt, es handele sich hier um eine Art Bespassungsaktion, deren Anspruch sie mit «Kindergarten» gleichsetzt. Sie scheint sich nicht ernstgenommen zu fühlen. Der kritische Ansatz der Künstlerin und des Künstlers, ihr Interesse an der Selbstermächtigung der Jugendlichen im Umgang mit der alltäglichen Kontrolle kann zum Start dieses Freizeitangebots im Jugendzentrum noch nicht deutlich werden.

\subsubsection{Szene 4: Annäherungen}

Draussen führt die Künstlerin die Jugendlichen zur Überwachungskamera am Kellereingang. Die Jugendlichen sollen ausgehend vom Gelände Bewegungen improvisieren. Tümay Kılınçel macht es vor: Sie geht die Treppe stampfend hinunter, bleibt stehen, springt hoch und greift dabei nach dem Geländer des Treppenabsatzes. Dort hängt sie, bevor sie sich fallen lässt und mit einer $180^{\circ}$-Drehung aufspringt, sodass sie die Treppe wieder vor sich hat und stampfend hinaufgeht.

«Thomas schaut zu, lässt sich aber nicht darauf ein und möchte lieber eine Zigarette rauchen. Tümay greift dies auf und schlägt vor, die Kamera während des Rauchens anzuschauen. Sie schlägt vor, dies als Choreografie zu interpretieren und eine feste Abfolge $z u$ entwickeln: Auftritt, Ankommen, hinsetzen, Zigarette drehen, anstecken, hochschauen, halten, zu Ende rauchen, aufstehen, Abgang.» (Feldnotiz BS_09_003_20200908,4) 
Der künstlerische Strategiewechsel bringt Erfolg: Die ersten Bewegungen vor der Kamera sind Thomas vertraut und stellen durch ihre Choreografie doch eine Ausnahme vom Alltag dar. Der ständige Blick in die Kamera macht dabei das Provozierende der Handlungen körperlich erfahrbar. Gleichzeitig werden Kılınçel \& Schaper Teil der Aufnahme. Es ist ein Moment gegenseitiger Annäherung (Deterritorialisierung): Die Künstlerinnen und Künstler kommen dem Wunsch des Jugendlichen nach und er lässt sich darauf ein, Teil der Kunst zu werden. Er und die umstehenden Jugendlichen erfahren, dass das Videoprojekt keine didaktische Planung hat, die 〈durchgezogen〉 werden muss, sondern dass es ihrem Rhythmus und ihrer Lust folgen wird (Reterritorialisierung).

\subsubsection{Szene 5: Koproduktion}

Im Verlauf des Tages kommen weitere Jugendliche hinzu. Auch Claire lässt sich später darauf ein, als ihre Freundin Vanessa ankommt, die Lust aufs Mitmachen hat. Die Künstlerin und der Künstler animieren sie mit der Zeit zu immer absurderen Szenen vor den Überwachungskameras, so etwa eine Serie von Auftritten, die sie nacheinander vor der Kamera am Eingang des Hauses aufführen: Kim tritt aus dem Haus und lässt Tee aus einer Kanne auf den Boden laufen, während sie fest in die Kamera blickt. Kurz darauf folgt Claire, die eine Handschaufel voll Mehl in die Luft schwingt und einen feinen Mehlregen über sich und den Hof verteilt. Anschliessend kommt Vanessa mit einem Stubenbesen, kehrt einmal links und rechts und geht wieder ab, die Kamera immer fest im Blick (vgl. Feldnotiz BS_09_003_20200908, 6). Nach und nach kommen die Jugendlichen selbst auf Ideen. Die Arbeit vor den Überwachungskameras wird zur Koproduktion: Im Wechsel mit Impulsen der Künstlerin und des Künstlers entstehen die nächsten Momente: Claire und Vanessa setzen Regenschirme als Blickschutz ein, Kim kämmt sich die Haare mit einem Handfeger, Thomas steht mit einer brennenden Wunderkerze still vor der Überwachungskamera. 
«Ein Junge mit Badeschlappen, kurzer Hose in grauem Tarnmuster und Pulli kommt dazu. Claire begrüßt ihn: 〈Hi, Sascha! Wir spielen gerade〉, kichert sie und steckt Vanessa damit an. Beide posieren und springen in verschiedene Positionen, während sie immer in die Sicherheitskameras gucken. Als [die Leiterin] hinter Sascha auf das Gelände kommt, erkenne ich den Jungen. Dann waren sie wohl fertig mit der Anhörung auf der Polizei. Sascha wirkt etwas geknickt, weil er bei dem Videoprojekt mitmachen wollte und nun so viel verpasst hat. Tümay und Cornelius spannen ihn daher sofort ein» (Feldnotiz BS_09_003_20200908, 7).

Durch die Koproduktion von Künstlerin, Künstler und Jugendlichen wird deutlich, dass die anfängliche Skepsis auf vielen Ebenen verortet war: Aufgewühlt sein durch die polizeiliche Vorladung eines Freundes, fehlende Erfahrung mit kreativen Kunstprojekten, unverständliche Erläuterungen der Künstlerin und des Künstlers zum Vorhaben, ein ungeklärtes Verhältnis zwischen Teilnehmenden, der Künstlerin und dem Künstler. Wichtig für die Jugendlichen war es zudem, an den Reaktionen ihrer Bezugspersonen zu sehen, dass sie sich trauen dürfen - die motivierte Mitarbeiterin, der neugierige Praktikant, die gute Freundin holten Claire aus ihrer Verweigerungshaltung heraus. Die Künstlerin und der Künstler hätten dies wohl alleine nicht geschafft, wussten aber, die Hinzukommende[n] sofort zu integrieren, sobald die Blockade sich auflöste. Nicht zu übersehen war zudem die Tatsache, dass es sich um ein offenes Angebot handelte: Die Jugendlichen durften selbst entscheiden, ob und wie lange sie teilnehmen wollten. Der Wunsch nach Zeit am Handy war (genauso wie das Rauchen) sowohl in die Aktionen vor der Kamera integrierbar als auch als Rückzugsmoment willkommen.

\subsubsection{Szene 6: Zerkratzung}

Bis zum Tag der Zusammenarbeit hatten die Künstlerin und der Künstler noch nicht klären können, wie sie schliesslich an die Aufzeichnungen der Kameras gelangen würden. Die Arbeit vor den Überwachungskameras war also auch eine Wette darauf, wie das Material am Ende aussehen würde 
(Qualität, Bildausschnitt) und ob die Kameras auch tatsächlich aufzeichneten. Das Ergebnis ist ambivalent: Einerseits kann die Anfrage als Teil einer künstlerischen Intervention gesehen werden, die der Verwaltung vermittelte, dass die Überwachung vom Residenzteam kritisch gesehen wurde. Andererseits zeigte sich bei der Sichtung des schliesslich von der Gemeinde übergebenen Bildmaterials, dass die Linse einer der Kameras so zerkratzt war, dass die überwachte Treppe nicht mehr zu sehen war. Der beauftragten Firma war das bis dahin nicht aufgefallen. Während die Künstlerin und der Künstler also mit ihrer Aktion stigmatisierende Überwachung thematisieren wollten, könnten sie letztlich dazu beigetragen haben, dass diese nun langfristig besser durchgeführt werden kann. An der zerkratzten $\mathrm{Ka}-$ meralinse zeigt sich auch ein weiteres Mal: Das Residenzteam kennt wegen seiner vergleichsweise kurzfristigen Aufenthaltszeit in Schotten nicht alle Ebenen der lokalen Konflikte und Denkweisen. Vor und während des Workshops äusserten die Künstlerin und der Künstler mehrfach, dass sie nicht sicher seien, ob die Jugendlichen wirklich verstünden, was sie von ihnen wollten. Das Zerkratzen der Linse zeigt jedoch, dass durchaus ein Problembewusstsein für die Überwachung vorhanden ist und bereits zu eigenmächtigen Massnahmen gegriffen wurde - wenngleich wohl nicht von den teilnehmenden Jugendlichen, die die Künstlerin und der Künstler darauf hingewiesen hätten, dass die Aktionen vor dieser Kamera keinen Sinn haben. In diesem Sinne knüpft die Arbeit von Kılınçel \& Schaper unintendiert an Widerstandsstrategien der Jugendlichen an. Jedoch stellen das Zerkratzen und das Zurückschauen in die Kamera zwei sehr unterschiedliche Wege des Umgangs dar. Der Weg der Künstlerin und des Künstlers wird als kulturelle Bildung gerahmt und von der Gemeinde entsprechend unterstützt. Er stösst potenziell sowohl bei den Jugendlichen als auch der Gemeinde eine Auseinandersetzung mit der Überwachung und den Selbstund Fremdbildern der Jugendlichen an. Der Weg des Zerkratzens dagegen ist ein subversiver Akt, der besonders dadurch wirksam wurde, dass er sich der Aufmerksamkeit so lange entziehen konnte. Er setzt nicht auf eine potenzielle Verbesserung, sondern schafft Fakten, die den Wunsch nach Entzug und Freiraum sofort herstellen. 
Im Rahmen ihrer Residenz traten Kllınçel \& Schaper temporär in die Assemblage des Jugendzentrums hinein. Sie veränderten sie nicht grundlegend, gaben aber den Jugendlichen ein erweitertes Handlungsspektrum mit auf den Weg, indem sie mit ihrer Kunst soziokulturelle Bedingungen postdigitaler Gesellschaften thematisieren (vgl. Herlitz und Zahn 2019, 2).

\section{Fazit}

Im Rahmen der künstlerischen Residenz in Schotten verschränkten sich die Interessen von Kılınçel \& Schaper temporär mit denen des Jugendzentrums. Dabei zeigte ihre Arbeit Parallelen zum Selbstverständnis der Organisation (sozialräumliche Orientierung, Bildungsarbeit, inklusive Jugendhilfe), gab aber auch Neuem Raum (Ermunterung der Jugendlichen zum kritischen Umgang mit der Überwachung). Damit wurde keine grundlegende Veränderung des stigmatisierenden Verhältnisses von Jugendlichen und Gemeinde angestossen, aber die Künstlerin und der Künstler eröffneten den Jugendlichen mithilfe künstlerischer Verfahrensweisen, die digitalen und physischen Raum verbinden, ein kreativ erweitertes Handlungsspektrum über die Zerstörung der Überwachungsapparate hinaus. Die Künstlerin glaubt an die Macht der Kunst, den Alltag verändern zu können. Die Jugendlichen orientieren sich an Menschen, die ihnen ohne Vorverurteilung entgegentreten, ihnen die Freiheit geben, die sie brauchen, ihnen aber auch Ideen geben, sich auf eine Weise mit ihrer Umwelt (hier der Überwachung ihrer Plätze) auseinanderzusetzen, die legal und sogar erwünscht ist. Die erwachsenen Künstlerinnen und Künstler und die Jugendlichen treffen sich dort, wo verstanden wird, das aktiv Widerständische, die anerkennende Zuwendung und ein gewisses Mass an Freiheit beider Seiten zu einem gemeinsamen Ziel - reterritorialisieren - verbinden zu können.

Mit diesem geschärften Blick erscheint die Residenz nicht nur als Aufblätterung der Vielfalt der Gemeinde, sondern auch als Möglichkeit zur Deterritorialisierung lokaler Narrative und der damit verbundenen Machtfigurationen. Indem die entstandene Website viel zeigt und wenig einordnet, ermöglicht sie Perspektiven abseits gewohnter Ordnungen. Je nach dem programmiert-zufälligen Aufbau der Seite im Moment des Besuchs wird vielleicht neben einer Gruppenaktion der Jugendlichen die 
Audio-Aufnahme eines Interviews platziert, das aus einem ganz anderen Kontext stammt. Oder das Video mit dem 〈Mehlwurf〉 erscheint zufällig neben dem Firmenschild einer Bäckerei und wirkt wie die augenzwinkernde Fortführung der Szene auf dem Schild.
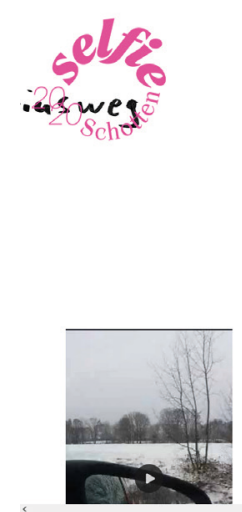
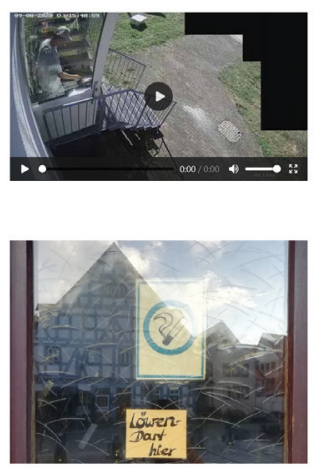

Abb. 3: Geschäftstafel einer Bäckerei in Schotten und Mehlwerfen am Jugendzentrum (Ausschnitt aus der Website schotten2020.de; 21.01.2022).

In ihrer digitalen Assemblage lösen Kılınçel \& Schaper die Selbstaufnahmen der Jugendlichen aus dem Kontext des Jugendzentrums und den damit verbundenen Deutungsmustern, um ihnen im Kontext anderer Selbstaufnahmen aus der Gemeinde die Chance auf neue Interpretationsansätze durch die Betrachtenden zu eröffnen. Auf diese Weise können partizipative künstlerische Projekte mit Jugendlichen Machtfigurationen zwar nicht allein und sofort verändern, aber sie können sie befragen, einem lokalen Publikum die Entwicklung neuer Sichtweisen ermöglichen und den Jugendlichen alternative Handlungsangebote für ein kreatives Aushandeln von Freiräumen an die Hand geben. So werden Impulse für die Persönlichkeitsentwicklung gegeben, dementsprechend findet im Videoprojekt kulturelle Bildung im oben skizzierten Verständnis statt.

In der Begleitung künstlerischer Residenzen in ländlichen Räumen erscheinen sozialer und künstlerischer Prozess als gleichberechtigte Teile einer gegenseitigen kulturellen Bildung, die Künstlerinnen, Künstler und Teilnehmende gemeinsam durchlaufen. Denn nicht nur die Jugendlichen machten in diesem Beispiel neue Erfahrungen, auch Künstlerin und 
Künstler mussten lernen, ihre künstlerischen Interessen mit unterschiedlichsten Partnerinnen und Partnern auszuhandeln und in Einklang mit deren Interessenlagen zu bringen. Mit ihrer Arbeit agieren Kılınçel \& Schaper temporär in einem lokalen Jugendzentrum und sind herausgefordert, sich innerhalb persönlicher, pädagogischer, machtvoller und machtkritischer sowie künstlerisch-ästhetischer Spannungsfelder zu verorten. Dabei zeigt sich, "dass sich pädagogisches Handeln und künstlerisches Handeln in Bildungskontexten wechselseitig bedingen» (Hinz 2018, 182) und sich innerhalb von Machtfigurationen bewegen. Künstlerische Verfahrensweisen führen ein Potenzial mit sich, diese kritisch auf ästhetische Weise zu befragen (vgl. Westphal 2018). Die Wechselwirkungen zwischen Konzept, Prozess und Produkt gilt es im weiteren Verlauf des Forschungsprojekts $\left\langle\mathrm{DO} \_\mathrm{KiL}\right\rangle$ in allen begleiteten Residenzen herauszuarbeiten und auf ihre machtkritischen Potenziale zu überprüfen.

\section{Literatur}

Bauer, Teresa. 2020. «Partizipation ermöglichen. Die Bedeutung sozialräumlicher Beteiligungsprozesse in der offenen Jugendarbeit». In Entgrenzte Jugend - offene Jugendarbeit: Jugend ermöglichen im 21. Jahrhundert, 1. Aufl., herausgegeben von Richard Krisch, und Wolfgang Schröer, 26-39. Weinheim, Grünwald: Beltz Juventa.

Bohnsack, Ralf, Iris Nentwig-Gesemann, und Arnd-Michael Nohl. 2013. «Einleitung: Die dokumentarische Methode und ihre Forschungspraxis». In Die dokumentarische Methode und ihre Forschungspraxis: Grundlagen qualitativer Sozialforschung, 3. akt. Aufl. herausgegeben von Ralf Bohnsack, Iris NentwigGesemann, und Arnd-Michael Nohl, 9-32. Wiesbaden: Springer VS. https:// doi.org/ 10.1007/978-3-531-19895-8.

Deinet, Ulrich, und Richard Krisch. 2021. «Das sozialräumliche Konzept in der Offenen Kinder- und Jugendarbeit». In Handbuch Offene Kinder- und Jugendarbeit, herausgegeben von Ulrich Deinet, Benedikt Sturzenhecker, Larissa von Schwanenflügel, und Moritz Schwerthelm, 1055-68. Wiesbaden: Springer VS. https://doi.org/10.1007/978-3-658-22563-6.

Deleuze, Gilles, und Félix Guattari. 1987. A Thousand Plateaus: Capitalism and Schizophrenia. Minneapolis, London: University of Minnesota Press.

Eckardt, Frank. 2018. «Rurbanität als Sozialraum: Jugendliche in der Thüringer Peripherie und die Verhandlung eines urbanen Lebensstils». In Rurbane Landschaften: Perspektiven des Ruralen in einer urbanisierten Welt, herausgegeben von Sigrun Langner, und Maria Frölich-Kulik, 189-202. Rurale Topografien Band 7. Bielefeld: transcript. https://doi.org/10.14361/9783839444283-fm. 
FLUX - Netzwerk Theater und Schule. o. J. «Residenzen». 21. April 2021. https:// flux-hessen.de/residenzen/.

Generaldirektion der Europäischen Kommission für Bildung, Jugend, Sport und Kultur. 2016. «Policy handbook on artists' residencies: European agenda for culture: work plan for culture 2011-2014». Luxembourg: Publications Office of the European Union. https://doi.org/10.2766/199924.

Herlitz, Lea, und Manuel Zahn. 2019. «Bildungstheoretische Potentiale postdigitaler Ästhetiken - Eine methodologische Annäherung». KULTURELLE BILDUNG ONLINE. https://doi.org/10.25529/92552.526.

Hinz, Melanie. 2018. «Wo ist denn hier eigentlich die Pädagogik? Über die Spezifik sozial-künstlerischen Handelns in Bildungskontexten». In Zwischen Kunst und Bildung: Theorie, Vermittlung, Forschung in zeitgenössischer Theater-, Tanzund Performancekunst, 1. Aufl., herausgegeben von Kristin Westphal, Teresa Bogerts, Mareike Uhl, und Ilona Sauer, 169-89. Theater | Tanz | Performance Band 3. Oberhausen: ATHENA.

Imbusch, Peter. 2007. «Macht: Dimensionen und Perspektiven eines Phänomens». In Journalismustheorie: Next Generation. Soziologische Grundlegung und theoretische Innovation, 1. Aufl., herausgegeben von Klaus-Dieter Altmeppen, Thomas Hanitzsch, und Carsten Schlüter, 395-419. Wiesbaden: VS Verlag. für Sozialwissenschaft. https://doi.org/10.1007/978-3-531-90401-6.

Kemper, Raimund, und Christian Reutlinger. Hrsg. 2015. Umkämpfter öffentlicher Raum. Wiesbaden: Springer VS. https://doi.org/10.1007/978-3-658-03437-5.

Keuchel, Susanne, und Bünyamin Werker, Hrsg. 2018. Künstlerisch-pädagogische Weiterbildungen für Kunst- und Kulturschaffende: Innovative Ansätze und Erkenntnisse. Band I Praxis. Wiesbaden: Springer VS. https://doi.org/10.1007/9783-658-20711-3.

Kilınçel, Tümay, und Cornelius Schaper. 2020. "Schotten2020». http://www. schotten2020.de/.

Kolleck, Nina, und Martin Büdel. 2020. «Kulturelle Bildung in ländlichen Räumen: Vorstellung der Forschungsvorhaben der BMBF-Förderrichtlinie». KULTURELLE BILDUNG ONLINE. https://doi.org/10.25529/92552.558.

Mandel, Birgit. 2016. «Kulturelle Vielfalt der Einwanderungsgesellschaft als Motor für Transformation des Kulturbetriebs in Deutschland». In Jahrbuch für Kulturpolitik 2015/2016: Thema: Transformatorische Kulturpolitik, herausgegebenvon Norbert Sievers, Patrick S. Föhl und Tobias J. Knoblich, 391-97. Bielefeld: transcript. https://doi.org/10.1515/9783839435120.

Oelkers, Nina, und Sascha Schierz. 2019. «Zur Interdependenz von Ländlichkeit und Sicherheit im «doing ruralityı». In Sicherheitsmentalitäten im ländlichen Raum, herausgegeben von Daniela Klimke, Nina Oelkers, und Martin K. W. Schweer, 83-106. Wiesbaden: Springer VS. https://doi.org/10.1007/978-3-65815118-8.

Rat für Kulturelle Bildung, Hrsg. 2015. Zur Sache: Kulturelle Bildung: Gegenstände, Praktiken und Felder. Essen: Rat für Kulturelle Bildung e.V. 
Rat für Kulturelle Bildung, Hrsg. 2014. Schön, dass ihr da seid: Kulturelle Bildung: Teilhabe und Zugänge. Essen: Rat für Kulturelle Bildung e.V.

Seitz, William Chapin. 1961. The Art of Assemblage. The Museum of Modern Art, New York: Doubleday. https://assets.moma.org/documents/moma_catalogue_1880_300062228.pdf.

Ullrich, Wolfgang. 2019. Selfies: Digitale Bildkulturen. Allgemeines Programm Sachbuch. Berlin: Wagenbach.

Waburg, Wiebke, Kristin Westphal, Micha Kranixfeld, und Barbara Sterzenbach. 2022. «Künstlerische Residenzen als Impulse in ländlichen Räumen? Theoretische und empirische Annäherungen». In Forschung zu kultureller Bildung in ländlichen Räumen: Methoden, Theorien und erste Befunde, herausgegeben von Martin Büdel, Nina Kolleck, und Jenny Nolting, 263-79. Weinheim, Basel: Belz Juventa.

Westphal, Kristin. 2018. "Teilhabe und Kritik als eine ästhetische Raumpraxis am Beispiel Theater und Schule». In VER_HANDELN: Begegnungen im öffentlichen Raum der Kunst — Skulptur Projekte 2017, herausgegeben von Birgit Engel, Ingrid Fisch, Stefan Hölscher, und Anna-Lena Treese, 76-89. Didaktische Logiken des Unbestimmten Band 3. München: kopaed. https://www.kunstakademie-muenster.de/fileadmin/media/Website_2018/kunst_wissenschaftliche_professuren_mitarbeiter/Birgit_Engel/PDFs/kunstakademie_band3_ klein_markierungen.pdf. 